\title{
First Detection of the Simplest Organic Acid in a Protoplanetary Disk *
}

\author{
Cécile Favre $^{1}$ (D), Davide Fedele ${ }^{1}$, Dmitry Semenov ${ }^{2,3}$, Sergey Parfenov ${ }^{4}$, Claudio Codella ${ }^{1,5}$ (i), Cecilia Ceccarelli ${ }^{5}$ (D), \\ Edwin A. Bergin ${ }^{6}$ (D) Edwige Chapillon ${ }^{7,8}$, Leonardo Testi ${ }^{1,9,10}\left(\mathbb{D}\right.$, Franck Hersant ${ }^{7}$, Bertrand Lefloch ${ }^{5}$, Francesco Fontani ${ }^{1}$ (D), \\ Geoffrey A. Blake ${ }^{11}$ (D) L. Ilsedore Cleeves ${ }^{12}$, Chunhua $\mathrm{Qi}^{12}$, Kamber R. Schwarz ${ }^{6}$ (D), and Vianney Taquet ${ }^{1}$ (D) \\ ${ }_{1}^{1}$ INAF-Osservatorio Astrofisico di Arcetri, Largo E. Fermi 5, I-50125, Florence, Italy; cfavre@ arcetri.astro.it \\ ${ }^{2}$ Max Planck Institute for Astronomy, Knigstuhl 17, D-69117 Heidelberg, Germany \\ ${ }^{3}$ Department of Chemistry, Ludwig Maximilian University, Butenandtstr. 5-13, D-81377 Munich, Germany \\ ${ }^{4}$ Ural Federal University, 51 Lenin Str., Ekaterinburg 620000, Russia \\ ${ }^{5}$ Univ. Grenoble Alpes, CNRS, IPAG, F-38000 Grenoble, France \\ ${ }^{6}$ Department of Astronomy, University of Michigan, 1085 South University Avenue, Ann Arbor, Michigan 48109, USA \\ ${ }^{7}$ Laboratoire d'astrophysique de Bordeaux, Univ. Bordeaux, CNRS, B18N, alle Geoffroy Saint- Hilaire, F-33615 Pessac, France \\ ${ }^{8}$ IRAM, 300 Rue de la Piscine, F-38046 Saint Martin d'Hères, France \\ ${ }^{9}$ European Southern Observatory, Karl-Schwarzschild-Str. 2, D-85748 Garching, Germany \\ ${ }^{10}$ Excellence Cluster Universe, Boltzmannstr. 2, D-85748 Garching, Germany \\ ${ }^{11}$ Division of Geological and Planetary Sciences, MC 150-21, California Institute of Technology, 1200 East California Boulevard, Pasadena, California 91125, USA \\ ${ }^{12}$ Harvard-Smithsonian Center for Astrophysics, 60 Garden Street, Cambridge, Massachusetts 02138, USA \\ Received 2018 April 23; revised 2018 June 20; accepted 2018 June 24; published 2018 July 16
}

\begin{abstract}
The formation of asteroids, comets, and planets occurs in the interior of protoplanetary disks during the early phase of star formation. Consequently, the chemical composition of the disk might shape the properties of the emerging planetary system. In this context, it is crucial to understand whether and what organic molecules are synthesized in the disk. In this Letter, we report the first detection of formic acid $(\mathrm{HCOOH})$ toward the TW Hydrae protoplanetary disk. The observations of the trans-HCOOH $6_{(1,6)-5(1,5)}$ transition were carried out at $129 \mathrm{GHz}$ with Atacama Large Millimeter/Submillimeter Array (ALMA). We measured a disk-averaged gas-phase t-HCOOH column density of $\sim(2-4) \times 10^{12} \mathrm{~cm}^{-2}$, namely as large as that of methanol. HCOOH is the first organic molecule containing two oxygen atoms detected in a protoplanetary disk, a proof that organic chemistry is very active, albeit difficult to observe, in these objects. Specifically, this simplest acid stands as the basis for synthesis of more complex carboxylic acids used by life on Earth.
\end{abstract}

Key words: astrochemistry - ISM: molecules - protoplanetary disks - radio lines: ISM stars: individual (TW Hya)

Supporting material: tar.gz file

\section{Introduction}

Life on Earth is based on different combinations of a relatively small number of key organic constituents, synthesized from simpler building-blocks including amino acids, phosphates, esters, organic acids, sugars, and alcohols. Some of these prebiotic molecules have been discovered in Solartype star-forming regions (Cazaux et al. 2003; Jørgensen et al. 2012; Kahane et al. 2013) as well as in meteoritic (Kvenvolden et al. 1970) and cometary (Elsila et al. 2010; Altwegg et al. 2016) material. Astronomers have long wondered whether the organic chemistry during the star and planet formation process is inherited by the planets and small bodies of the final planetary system, and what the key organic molecules are. In order to establish this missing link, it is mandatory to understand how organic chemistry evolves during the protoplanetary disk phase, which is the last step before the planet formation. So far, however, only about twenty molecules have been detected in protoplanetary disks (Dutrey et al. 2014). Among them are small hydrocarbons, such as $\mathrm{c}-\mathrm{C}_{3} \mathrm{H}_{2}$ (Qi et al. 2013b; Bergin et al. 2016), and

\footnotetext{
* This Letter makes use of the following ALMA data: ADS/JAO ALMA\#2015.1.00845.S (PI C. Favre). ALMA is a partnership of ESO (representing its member states), NSF (USA) and NINS (Japan), together with NRC (Canada), NSC and ASIAA (Taiwan), and KASI (Republic of Korea), in cooperation with the Republic of Chile. The Joint ALMA Observatory is operated by ESO, AUI/NRAO and NAOJ.
}

cyanides $\mathrm{HC}_{3} \mathrm{~N}$ and $\mathrm{CH}_{3} \mathrm{CN}$ (Chapillon et al. 2012; Öberg et al. 2015; Bergner et al. 2018), as well as two organic O-bearing molecules, methanol, $\mathrm{CH}_{3} \mathrm{OH}$ (Walsh et al. 2016), and formaldehyde, $\mathrm{H}_{2} \mathrm{CO}(\mathrm{Qi}$ et al. 2013a; Loomis et al. 2015; Carney et al. 2017; Öberg et al. 2017), believed to be the first step toward a complex organic chemistry. The search for relatively large molecules in protoplanetary disks remains challenging (Walsh et al. 2014): high sensitivity and resolution (spatial and spectral) are required. The unprecedented sensitivity of the Atacama Large Millimeter/ Submillimeter Array (ALMA) makes this instrument the most suitable for such a study.

In this Letter, we focus on formic acid ( $\mathrm{HCOOH})$, a key organic molecule as the carboxyl group $(\mathrm{C}(=\mathrm{O}) \mathrm{OH})$ is one of the main functional groups of amino acids (the structural units of proteins). Indeed, this species is involved in a chemical route leading to glycine, the simplest amino acid (e.g., see Basiuk 2001; Redondo et al. 2015). $\mathrm{HCOOH}$ is unambiguously detected toward both high- and low-mass star-forming regions (e.g., see Liu et al. 2002; Lefloch et al. 2017). Here, we report the first detection of $\mathrm{HCOOH}$ with ALMA toward the protoplanetary disk surrounding the closest (59 pc, Gaia Collaboration et al. 2016) Solar-type young star, TW Hya. TW Hya is a relatively old (3-10 Myr) T Tauri star of about $0.7 M_{\odot}$, which is surrounded by a gasrich disk, with a mass that is $\geqslant 0.006 M_{\odot}$ (Bergin et al. 2013; 
Trapman et al. 2017) and which shows prominent rings and gaps in gas and dust emission, perhaps a signature of ongoing planet formation. Notably, combined HD and CO observations toward TW Hya suggest that a substantial fraction of carbon reservoir is absent from the gas phase and could be stored in organic species, possibly in relatively complex ones (Favre et al. 2013; Schwarz et al. 2016; Zhang et al. 2017). This detection of a new class of organic species has important consequences, because a relatively high, observationally derived abundance of $\mathrm{HCOOH}$, along with that $\mathrm{CH}_{3} \mathrm{OH}$ $\left(\chi \sim 3 \times 10^{-12}-4 \times 10^{-11}\right.$, see Walsh et al. 2016), likely imply a rich organic chemistry in protoplanetary disks at the epoch of planet formation.

In Sections 2, we describe our observations. Results and analysis are given in Section 3. Physico-chemical modeling and further discussion are presented in Section 4.

\section{Observations and Data Reduction}

In this Letter, we focus on the trans-HCOOH $6_{(1,6)-5(1,5)}$ transition (i.e., the $\mathrm{OH}$ functional group is on the opposing side to the single $\mathrm{C}-\mathrm{H}$ bond) emitting at $129671.82 \mathrm{MHz}$. We used the spectroscopic data parameters from Kuze et al. (1982) that are available at the Cologne Database for Molecular Spectroscopy line catalog (Müller et al. 2005). This transition has an appropriate low upper-state energy level $(\sim 25 \mathrm{~K})$ and high line strength $\left(\sim 12 \mathrm{D}^{2}\right)$, making it ideal for searching for this molecule in the gas with a temperature range of about $10-40 \mathrm{~K}$, which is typical for the outer regions of protoplanetary disks surrounding young Sun-like stars like TW Hya. In addition, we note that six lines from the cis conformer, c-HCOOH, at $131 \mathrm{GHz}$ were also covered by the observations. However, the energy barrier to internal trans conversion to cis is very high $\left(\sim 1365 \mathrm{~cm}^{-1}\right.$, see Winnewisser et al. 2002), making their detection unlikely.

The observations were performed toward TW Hya on 2016 May 31 and on 2016 June 1 with 39 antennas and baselines from $15.1 \mathrm{~m}(6.5 \mathrm{k} \lambda)$ up to $713.1 \mathrm{~m}(310.0 \mathrm{k} \lambda)$ with an on-source time of about 39 min with ALMA during Cycle 3. The phase-tracking center was $\alpha_{J 2000}=11^{\mathrm{h}} 01^{\mathrm{m}} 51^{\mathrm{s}} .875$, $\delta_{J 2000}=-34^{\circ} 42^{\prime} 17 !^{\prime \prime} 155$. The spectral setup consisted of (i) six spectral windows, each with 960 channels and a channel width used during the observations of $244.141 \mathrm{kHz}$ (about $0.6 \mathrm{~km} \mathrm{~s}^{-1}$ ) that covered about $1.4 \mathrm{GHz}$ between 129.538 and $132.405 \mathrm{GHz}$, and (ii) one spectral window, centered at $130.973 \mathrm{GHz}$, of $2 \mathrm{GHz}$ bandwidth (128 channels each of $15.625 \mathrm{MHz}$ ) for continuum imaging. The quasars J1037-2934 and J1103-3251 were used as calibrators for bandpass and phase for these observations. Ganymede and Titan were used as flux calibrators for the observations performed in May and June, respectively. Data reduction and continuum subtraction were performed through the version 4.5 .3 of the Common Astronomy Software Applications (CASA). The continuum emission at $129 \mathrm{GHz}$ was self-calibrated with the solutions (gain tables) applied to the molecular data. In addition, and still in order to optimize the sensitivity, the data were cleaned using a "Natural" weighting. The resulting synthesized beam sizes are 1 !" $28 \times 1$." 00 at a P.A. $\sim-89^{\circ}$ and 1 !" $29 \times 1$ !" 02 at a P.A. of about $90^{\circ}$ for the continuum (see Figure 1) and line images, respectively.

\section{Results and Analysis}

\section{1. $t-\mathrm{HCOOH}$}

We detected the $129 \mathrm{GHz} \mathrm{HCOOH}$ line with a signal-tonoise ratio $(\mathrm{S} / \mathrm{N})$ of about 4 . Figure 2 shows the disk-averaged spectrum extracted from the native data set ${ }^{13}$ using an elliptical aperture of about 400 au to take into account uncertainties on the position and the spatial extent of the $\mathrm{HCOOH}$ emission. Figure 2 also displays the $\mathrm{HCOOH}$ spectrum resulting from the pixel spectral stacking method (assuming a Keplerian disk, see Yen et al. 2016), which independently strengthens our detection. Even though we have only one detected line, we can firmly confirm the identification of $\mathrm{HCOOH}$ because this is (i) the brightest line expected in the observed range, (ii) the same line was clearly detected in the Solar-like regions L1157-B1 and NGC1333-IRAS4A with the IRAM-30 m telescope as part of the Astrochemical Surveys At IRAM (ASAI) Large Program (see Lefloch et al. 2017, 2018), and (iii) two independent methods show a detection above the $4 \sigma$ level (see Figure 2).

To further enhance the spatial signal-to-noise ratio, sophisticated data processing has been performed (see Section 3.2).

\subsection{Data Processing: Keplerian Mask}

Assuming that the disk is in Keplerian rotation, one can significantly improve the $\mathrm{S} / \mathrm{N}$ by the use of a Keplerian mask (Carney et al. 2017; Salinas et al. 2017). More specifically, the latter selects the regions in the data where the signal is expected to emit, following a disk velocity pattern (including the rotational and the systemic velocities of the object) and a disk size.

The parameters that we used to define out TW Hya Keplerian mask for the $\mathrm{t}-\mathrm{HCOOH}$ emission are as follows:

1. stellar mass of $0.7 M_{\odot}$,

2. disk inclination of $7^{\circ}$,

3. disk position angle of $155^{\circ}$,

4. systemic velocity of $2.7 \mathrm{~km} \mathrm{~s}^{-1}$,

5. distance of $59 \mathrm{pc}$, consistent with the recent GAIA measurements (Gaia Collaboration et al. 2016),

6. outer radius of 400 au (to be consistent with the data).

The consistency of the mask that we used in this study has been checked on the $\mathrm{c}_{-} \mathrm{C}_{3} \mathrm{H}_{2}$ (3-2) line emission at $145089.6 \mathrm{MHz}$, which is clearly observed above the $12 \sigma$ level as part of our project (C. Favre et al. 2018, in preparation). After applying the above Keplerian mask to the $\mathrm{HCOOH}$ data, the $\mathrm{S} / \mathrm{N}$ was enhanced by a factor $\sim 5$. Note that to compute the "bootstrapped" noise, the same keplerian mask was applied to channels with no signal that were far away from the ones in which $\mathrm{HCOOH}$ is emitting (see Bergner et al. 2018). Then, the resulting $\mathrm{t}-\mathrm{HCOOH}$ emission was integrated over the line profile, from $v=0.9$ to $3.4 \mathrm{~km} \mathrm{~s}^{-1}$ and smoothed by a Gaussian kernel of $7^{\prime \prime} \times 7^{\prime \prime}$ to spatially enhance the $\mathrm{S} / \mathrm{N}$. Figure 3 displays the resulting smoothed disk-averaged $\mathrm{t}-\mathrm{HCOOH}$ spatial distribution (with a detection at a peak to noise ratio of $12 \sigma$ ), after applying the Keplerian mask.

It is important to note that the low spectral and spatial resolution of our observations allow us neither to disentangle the spatial structure of the emission nor to investigate the kinematics: (i) the line peak is slightly displaced with respect to

\footnotetext{
13 A FITS image of the t-HCOOH channel map is available in a tar.gz package.
} 


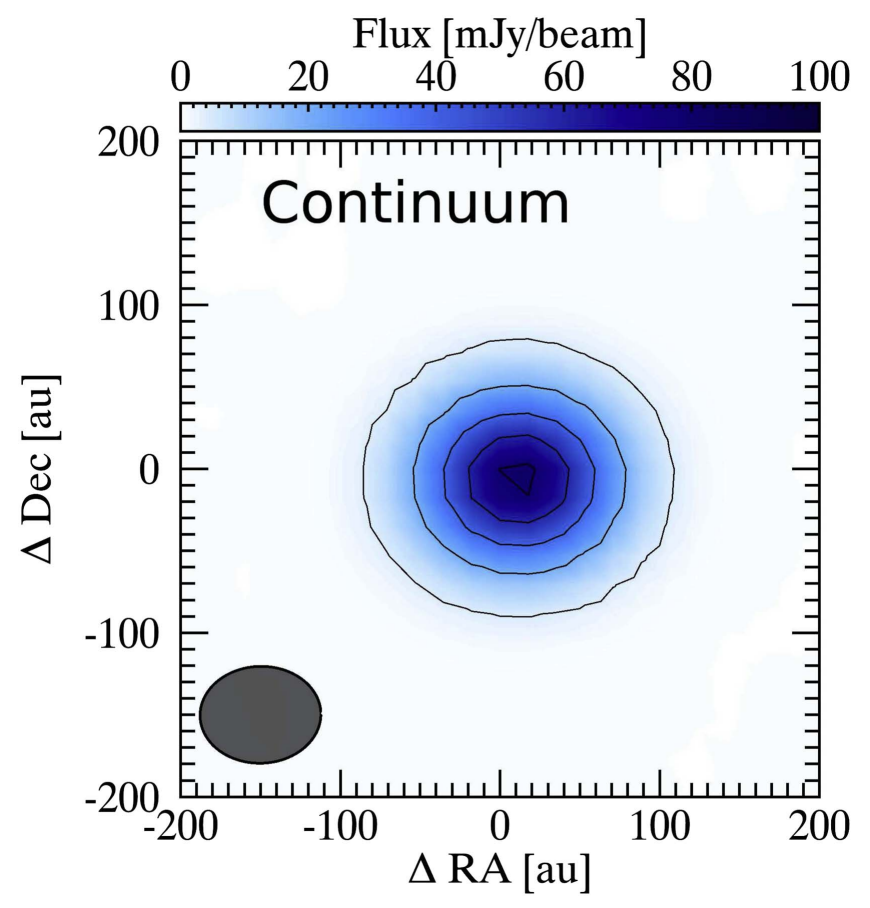

Figure 1. $129 \mathrm{GHz}$ continuum emission as observed with ALMA toward TW Hya. The contours and level step are at $14 \sigma$ (where $1 \sigma=1.4 \mathrm{mJy}^{\text {beam }}{ }^{-1}$ ).

the local standard of rest (LSR) velocity of the source $\left(2.86 \mathrm{~km} \mathrm{~s}^{-1}\right)$ by at most one channel, but this displacement is consistent with the spectral resolution $\left(0.6 \mathrm{~km} \mathrm{~s}^{-1}\right)$, and (ii) the low angular resolution observations, along with the convolution procedure, lead to at least 100 au of uncertainty regarding the spatial extent of $\mathrm{HCOOH}$.

\subsection{Column Densities and Relative Abundances}

The measured disk-averaged line integrated intensity over the line profile, $F=(89 \pm 12) \times 10^{-3} \mathrm{Jy} \mathrm{km} \mathrm{s}^{-1}$, corresponds to a $\mathrm{t}-\mathrm{HCOOH}$ source-averaged column density of $(2.9 \pm 1.3) \times$ $10^{12} \mathrm{~cm}^{-2}$ and $(3.5 \pm 0.8) \times 10^{12} \mathrm{~cm}^{-2}$ for an excitation temperature of 10 and $40 \mathrm{~K}$, respectively. The $10-40 \mathrm{~K}$ temperature range is the typical one where the molecular gas is expected to be emissive. Our observations suggest that $\mathrm{HCOOH}$ emission appears centrally peaked with extension beyond $200 \mathrm{au}$. Although our present low-resolution observations do not allow us to constrain exactly where formic acid is emitting within the TW Hya disk, one could simply assume that all organic O-bearing molecules emit within the same region if they share grain-surface formation chemistry. As methanol was previously detected toward TW Hya (Walsh et al. 2016), one can estimate the fraction of formic acid with respect to methanol, often believed to be a starting molecule from which more complex organics are synthesized, either in the gas-phase (Charnley et al. 1992; Balucani et al. 2015) or within the icy mantles of dust grains (Garrod \& Herbst 2006; Semenov \& Wiebe 2011). In that light, we obtain an average abundance t- $\mathrm{HCOOH} / \mathrm{CH}_{3} \mathrm{OH}$ ratio lower than and/or equal to 1 , which is about one order of magnitude higher than the ratio measured in comets (Biver et al. 2014). Here, we stress that this ratio likely suffers from the fact that we are not sampling the same spatial scales. Indeed, current methanol observations (Walsh et al. 2016) seem to indicate a more compact spatial distribution than our $\mathrm{t}-\mathrm{HCOOH}$ observations. Aside from the intrinsic limitations of our observations and that of methanol,
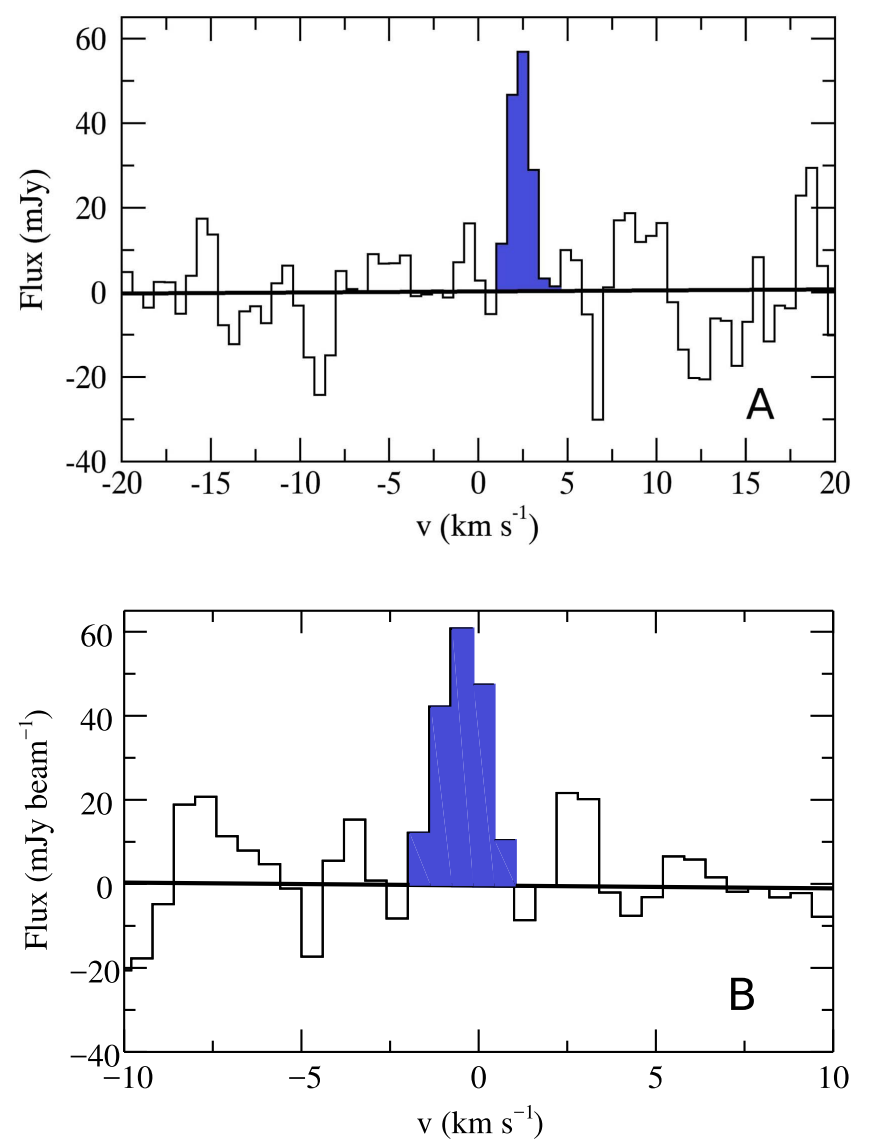

Figure 2. Panel (a): TW Hya disk-averaged $\mathrm{HCOOH}$ spectrum extracted from the original datacube within $400 \mathrm{au}$. Panel (b): TW Hya HCOOH spectrum resulting from the pixel spectral stacking method by Yen et al. (2016). The $\mathrm{S} / \mathrm{N}$ is $\sim 5$.

this difference in spatial distribution can originate from the different formation pathways.

\section{On the Production of $\mathrm{HCOOH}$}

From a chemical point of view, the chemistry leading to formic acid is more complex than that responsible for the methanol formation. Indeed, the latter is mostly the result of the hydrogenation of carbon monoxide on the surface of grains, forming $\mathrm{H}_{2} \mathrm{CO}$ and $\mathrm{CH}_{3} \mathrm{OH}$ from $\mathrm{CO}$ that is facilitated at low temperatures (about $20 \mathrm{~K}$ ), which allows the following: (i) $\mathrm{CO}$ molecules to freeze out onto the icy grain surfaces, (ii) hydrogen diffusion at the surface of grain mantles (prior to their evaporation), (iii) and consequently, hydrogen to tunnel through energy barriers which would be otherwise insurmountable (Watanabe \& Kouchi 2002; Rimola et al. 2014). Formic acid, on the contrary, cannot be a simple $\mathrm{H}$-addition process and has to be the result of reaction(s) involving polyatomic species, either on the grain surfaces or in the gas phase (Ioppolo et al. 2011; Skouteris et al. 2018).

\subsection{Modeling}

To better understand $\mathrm{HCOOH}$ chemistry across the disk, we used a state-of-the-art modeling suite (Parfenov et al. 2017) that includes a disk physical structure, radiative transfer, and chemical modeling that adequately describes the methanol observations in TW Hya disk (Walsh et al. 

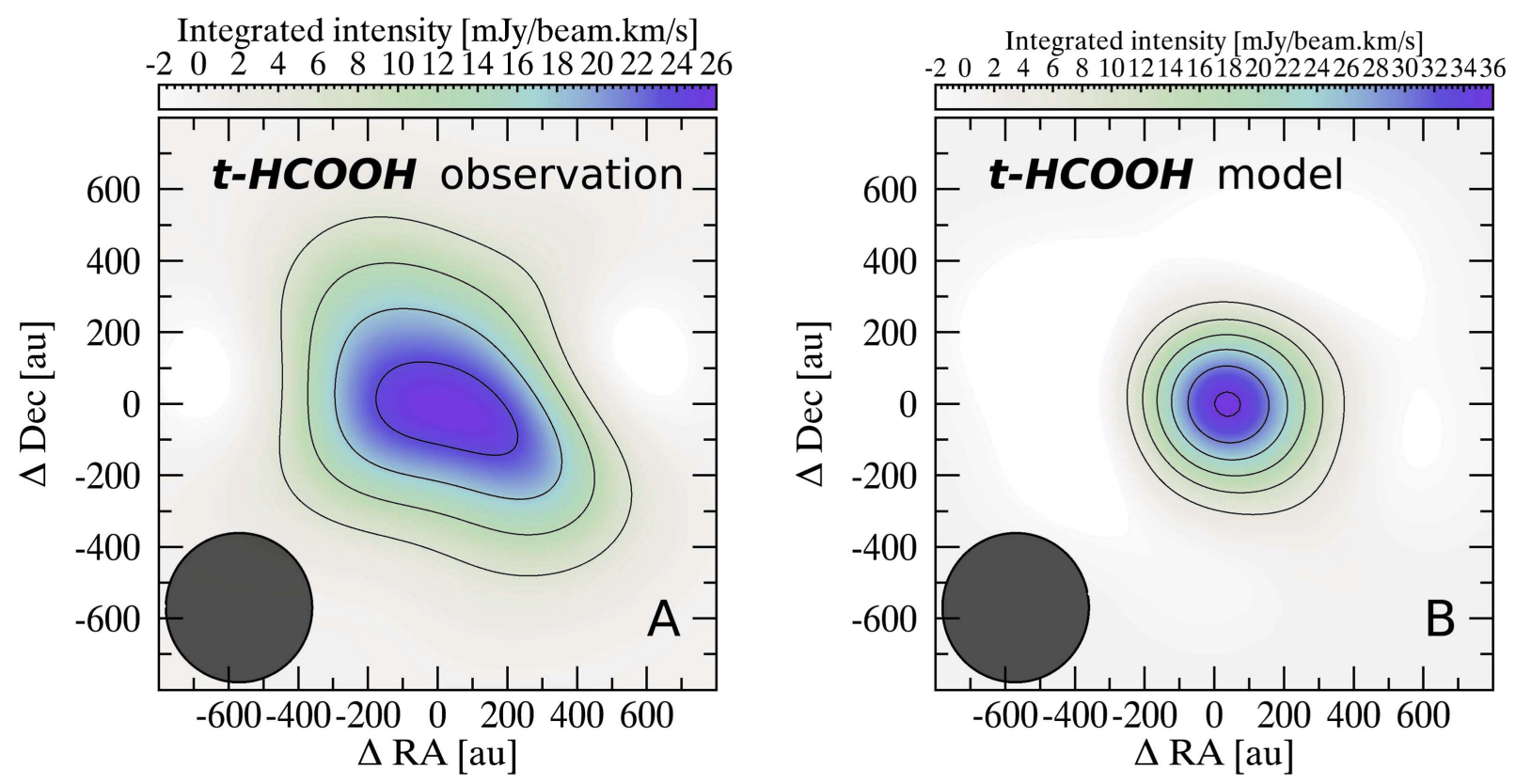

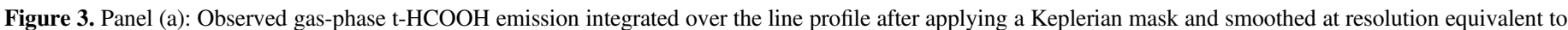

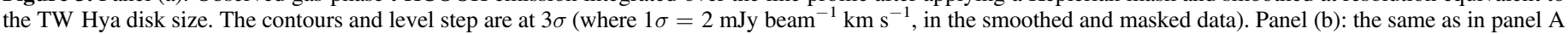
but model. The synthesized beam is shown in the bottom-left corner of panels (A) and (B).

2016) and within a factor 5 that of $\mathrm{CH}_{3} \mathrm{CN}$ by Loomis et al. (2018).

\subsubsection{Physical and Chemical Model of TW Hya Disk}

Our disk physical structure is computed using a thermochemical model by Gorti et al. (2011) for TW Hya. The disk spans 3.9-200 au radially and has separately computed gas and dust temperatures. An accretion rate of $10^{-9} M_{\odot} \mathrm{yr}^{-1}$ and a constant gas-to-dust mass ratio of 100 are assumed. The adopted parameters of the central star and the incident ultraviolet (UV) and X-ray radiation are representative of those observed in TW Hya. The star has a mass of $0.7 M_{\odot}$, a radius of $1 R_{\odot}$, and an effective temperature of $4200 \mathrm{~K}$. A far-UV (FUV) spectrum with a total FUV luminosity of $3 \times 10^{31} \mathrm{erg} \mathrm{s}^{-1}$ was used (Herczeg et al. 2002, 2004; Cleeves et al. 2014). The adopted X-ray spectrum, covering $0.1-10 \mathrm{keV}$, has a total X-ray luminosity of $1.6 \times 10^{30} \mathrm{erg} \mathrm{s}^{-1}$.

The chemical structure of the disk was computed with the public gas-grain ALCHEMIC code (see http://www.mpia.de/homes/ semenov/disk_chemistry_OSU08ggs_UV.zip, Semenov et al. 2010). The chemical network is based on the osu.2007 ratefile with recent updates to the reaction rates from Kinetic Database for Astrochemistry (Wakelam et al. 2012), and a high-temperature network (Harada et al. 2010; Semenov \& Wiebe 2011).

A standard cosmic-ray (CR) ionization rate was assumed to be $\zeta_{\mathrm{CR}}=1.3 \times 10^{-17} \mathrm{~s}^{-1}$, as it does not affect significantly the chemistry in comparison to the stellar X-rays. The FUV penetration into the disk is computed in a $1+1 \mathrm{D}$ approximation (Semenov \& Wiebe 2011) using the visual extinction $A_{V}$ in the direction toward the central star for the stellar FUV component, and the visual extinction $A^{\mathrm{IS}}$ in the vertical direction for the interstellar (IS) FUV component. To compute the X-ray ionization rate, we used the parametrization given by Armitage (2007) for the average X-ray photon energy of $3 \mathrm{keV}$. The self-shielding of $\mathrm{H}_{2}$ from photodissociation is calculated following the parametrization given in (Draine \& Bertoldi
Table 1

Initial Chemical Abundances

\begin{tabular}{lccc}
\hline \hline Species & Abundance & Species & Abundance \\
\hline ortho- $\mathrm{H}_{2}$ & $3.75(-1)$ & $\mathrm{S}$ & $9.14(-8)$ \\
para- $\mathrm{H}_{2}$ & $1.13(-1)$ & $\mathrm{Si}$ & $9.74(-9)$ \\
$\mathrm{He}$ & $9.75(-2)$ & $\mathrm{Fe}$ & $2.74(-9)$ \\
$\mathrm{O}$ & $1.80(-4)$ & $\mathrm{Na}$ & $2.25(-9)$ \\
$\mathrm{C}$ & $7.86(-5)$ & $\mathrm{Mg}$ & $1.09(-8)$ \\
$\mathrm{N}$ & $2.47(-5)$ & $\mathrm{Cl}$ & $1.00(-9)$ \\
$\mathrm{HD}$ & $1.55(-5)$ & $\mathrm{P}$ & $2.16(-10)$ \\
\hline
\end{tabular}

1996). The shielding of $\mathrm{CO}$ by dust grains, $\mathrm{H}_{2}$, and the $\mathrm{CO}$ selfshielding are calculated using a precomputed table (see Lee et al. 1996).

The gas-grain interactions include the sticking of neutral species and electrons to dust grains with $100 \%$ probability, and the desorption of ices by thermal, cosmic-ray particle (CRP), and UV-driven processes. We do not allow $\mathrm{H}_{2}$ to stick to grains. The uniformly sized, compact amorphous silicate particles with the radius $a_{d}=7 \mu \mathrm{m}$ are considered. This grain size represents grain surface per unit gas volume of the size ensemble used in the physical model. A low UV-photodesorption yield of $10^{-5}$ is adopted for all ices, based on the recent measurements (Bertin et al. 2016; Cruz-Diaz et al. 2016). Photodissociation processes of solid species are also taken into account (Garrod \& Herbst 2006; Semenov \& Wiebe 2011). A $1 \%$ probability for chemical desorption is assumed (Garrod et al. 2007). Surface recombination proceeds solely via Langmuir-Hinshelwood mechanism (Hasegawa et al. 1992), described by the standard rate equation approach. The ratio of diffusion to binding energy of surface species $E_{\text {diff }} / E_{b}$ is 0.4 , which is consistent with the recent laboratory studies (Cuppen et al. 2017). A "low metals", mainly atomic set of initial abundances (Lee et al. 1998; Semenov et al. 2010) was used (see Table 1). The adopted thermochemical disk physical 

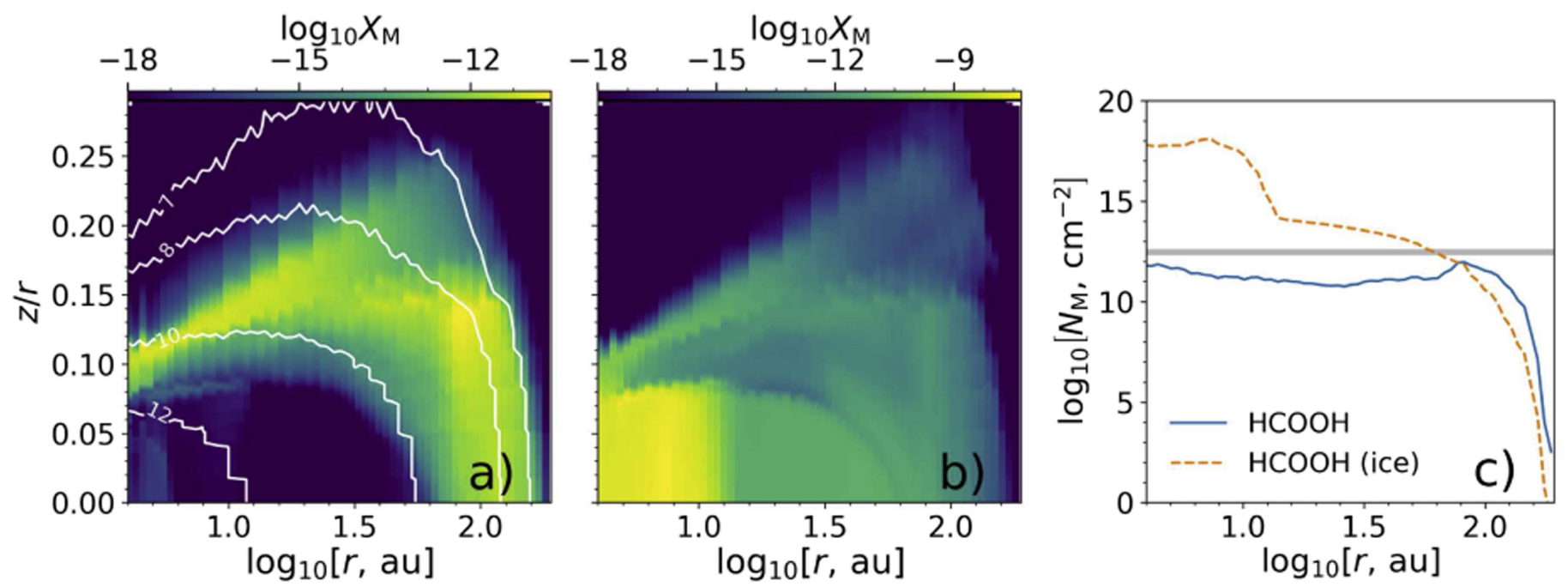

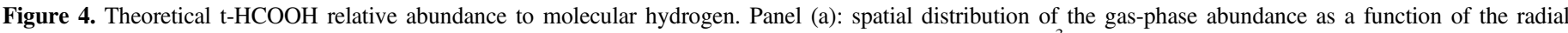

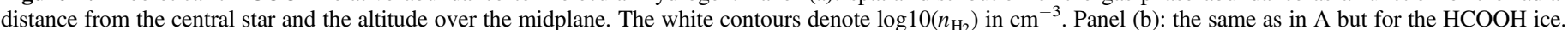

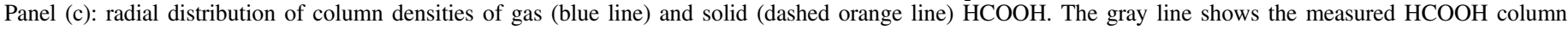
density. The predictions are based on our physical and chemical model (Parfenov et al. 2017, see Section 4).

model coupled with alchemic code was used to model the entire TW Hya disk chemical evolution over $t=1 \mathrm{Myr}$.

\subsubsection{Radiative Transfer Modeling}

To predict the $\mathrm{HCOOH}$ line emission from our disk model we used the three-dimensional Monte-Carlo code Line Modeling Engine (LIME; Brinch \& Hogerheijde 2010) with a setup similar to the one used by Parfenov et al. (2017) to reproduce methanol emission toward TW Hya by Walsh et al. (2016). The radiative transfer calculations were performed assuming local thermodynamic equilibrium (LTE). Non-LTE calculations could not be performed as the collisional rate coefficients for $\mathrm{HCOOH}$ are not available. Nonetheless, as shown in Figure 4 (see Panel (a)), $\mathrm{HCOOH}$ is abundant in the disk regions where the $\mathrm{H}_{2}$ density is relatively high $\left(n_{\mathrm{H} 2} \geqslant 10^{7} \mathrm{~cm}^{-3}\right)$ and assuming a "standard" collisional coefficient of $10^{-11}-10^{-10} \mathrm{~cm}^{-3} \mathrm{~s}^{-1}$, one might expect the line to be thermalized.

In order to simulate the spectral averaging within the ALMA channels, the synthetic image cubes produced by LIME (with a spectral resolution of $0.06 \mathrm{~km} \mathrm{~s}^{-1}$ ) were averaged along the spectral axis down to the resolution of $0.6 \mathrm{~km} \mathrm{~s}^{-1}$, which is the same as our observed data. These datacubes were then converted into visibilities using the simobserve task from the CASA package, using the same antenna positions as in the observations. Then noise was added to the visibilities using sm.setnoise and sm.setgain CASA tasks. Finally, the data were deconvolved and the image reconstructed via the clean task. The synthetic beam with the size of 1 ". $31 \times 0$. ". 98 at a position angle PA of $-79^{\circ} .6$, which well matches the observed beam. The rms noise level in the synthetic disk images is of about $1 \mathrm{mJy}^{-1}$ beam ${ }^{-1}$ per channel that is consistent with the observations at the original spatial resolution.

\subsection{Chemical Desorption as the Source of HCOOH in Disks?}

With the above model we are able to compute the evolution of the formic acid abundance over the age of TW Hya and thus to reconstruct its spatial distribution within the TW Hya protoplanetary disk (Figure 3(b)). Figure 4 shows the resulting modeled distribution of the $\mathrm{HCOOH}$ relative abundance to molecular hydrogen. Our physico-chemical modeling reproduces well the observed column densities within a factor 3 that is commensurate with the uncertainties (from both the observations and the model). In this model, it is assumed that $\mathrm{HCOOH}$ is formed by the recombination of $\mathrm{HCO}$ and $\mathrm{OH}$ radicals on the grain surfaces (Garrod 2008), and that a fraction of it $(1 \%)$ is injected into the gas phase through reactive desorption, because of the energy released by the reaction itself. In addition, some $\mathrm{HCOOH}$ molecules can be released from the ice mantles through CRPs heating of grains, CRPinduced UV-photodesorption, and direct UV desorption in the more irradiated outer disk region.

Interestingly enough, Skouteris et al. (2018) have recently shown that a new gas-phase scheme of reactions, involving ethanol as a parent molecule, can lead to the formation of formic acid. Using a preliminary 0D-model for disk-like conditions (Semenov et al. 2010), this new set of reactions does not seem to dominate the production of formic acid in disks. Nevertheless, this leads one to consider other gas-phase and/or grain-surface processes that may be worth studying in the future, as they could contribute to the observed distribution of formic acid.

\section{Conclusions}

In summary, we report the first detection of the simplest acid, $\mathrm{HCOOH}$, in the protoplanetary disk surrounding a Sun-like young star, TW Hya. Our finding implies that a rich organic chemistry, which can lead to larger organic molecules, likely takes place at the verge of planet formation in protoplanetary disks. Indeed, $\mathrm{HCOOH}$, together with methanol and formaldehyde (Walsh et al. 2014), are the most abundant complex molecules detected in protoplanetary disks so far. In the context of an interstellar-Earth connection, this shows that at least some of the bricks of prebiotic chemistry are already present in a protoplanetary disk expected to be similar to the Solar Nebula that formed our Solar System. Incidentally, our study strengthens the fact that observations of larger complex molecules in this environment remain challenging, even with ALMA and its 
unprecedented sensitivity. Finally, further improvements in our understanding of the formation of organic molecules, through both laboratory experiments and theory, will help us to target future exploration of the chemical richness of protoplanetary disks, in particular to better characterize where these molecules are expected to be located within the disk.

We thank the anonymous referee for useful comments. We are very grateful to Nathalie Brouillet for her comments on formic acid in the ISM. This work was supported by (i) the Italian Ministry of Education, Universities and Research, through the grant project SIR (RBSI14ZRHR), (ii) by the Italian Ministero dell'Istruzione, Universit e Ricerca through the grant Progetti Premiali 2012-iALMA (CUP C52I13000140001), (iii) funding from the European Research Council (ERC), project DOC (The Dawn of Organic Chemistry), contract 741002, and (iv) supported by the project PRIN-INAF 2016 The Cradle of Life-GENESIS-SKA (General Conditions in Early Planetary Systems for the rise of life with SKA). The work of S.P. was supported by Act 211 Government of the Russian Federation, contract \# 02. A03.21.0006 D.S. acknowledges support from the Heidelberg Institute of Theoretical Studies for the project "Chemical kinetics models and visualization tools: Bridging biology and astronomy."

\section{ORCID iDs}

Cécile Favre (1) https://orcid.org/0000-0002-5789-6931

Claudio Codella (1) https://orcid.org/0000-0003-1514-3074

Cecilia Ceccarelli (ib https://orcid.org/0000-0001-9664-6292

Edwin A. Bergin (1) https://orcid.org/0000-0003-4179-6394

Leonardo Testi (i) https://orcid.org/0000-0003-1859-3070

Francesco Fontani (1) https://orcid.org/0000-0003-0348-3418

Geoffrey A. Blake (i) https://orcid.org/0000-0003-0787-1610

Kamber R. Schwarz (1) https://orcid.org/0000-0002-6429-9457

Vianney Taquet (ib https://orcid.org/0000-0003-0407-7489

\section{References}

Altwegg, K., Balsiger, H., Bar-Nun, A., et al. 2016, SciA, 2, e1600285 Armitage, P. J. 2007, arXiv:astro-ph/0701485

Balucani, N., Ceccarelli, C., \& Taquet, V. 2015, MNRAS, 449, L16 Basiuk, V. A. 2001, JPCA, 105, 4252

Bergin, E. A., Cleeves, L. I., Gorti, U., et al. 2013, Natur, 493, 644

Bergin, E. A., Du, F., Cleeves, L. I., et al. 2016, ApJ, 831, 101

Bergner, J. B., Guzmán, V. G., Öberg, K. I., Loomis, R. A., \& Pegues, J. 2018, ApJ, 857, 69

Bertin, M., Romanzin, C., Doronin, M., et al. 2016, ApJL, 817, L12

Biver, N., Bockelée-Morvan, D., Debout, V., et al. 2014, A\&A, 566, L5

Brinch, C., \& Hogerheijde, M. R. 2010, A\&A, 523, A25

Carney, M. T., Hogerheijde, M. R., Loomis, R. A., et al. 2017, A\&A, 605, A21

Cazaux, S., Tielens, A. G. G. M., Ceccarelli, C., et al. 2003, ApJL, 593, L51

Chapillon, E., Dutrey, A., Guilloteau, S., et al. 2012, ApJ, 756, 58
Charnley, S. B., Tielens, A. G. G. M., \& Millar, T. J. 1992, ApJL, 399, L71 Cleeves, L. I., Bergin, E. A., \& Adams, F. C. 2014, ApJ, 794, 123

Cruz-Diaz, G. A., Martín-Doménech, R., Muñoz Caro, G. M., \& Chen, Y.-J. 2016, A\&A, 592, A68

Cuppen, H. M., Walsh, C., Lamberts, T., et al. 2017, SSRv, 212, 1

Draine, B. T., \& Bertoldi, F. 1996, ApJ, 468, 269

Dutrey, A., Semenov, D., Chapillon, E., et al. 2014, in Protostars and Planets VI, ed. H. Beuther et al. (Tucson, AZ: Univ. Arizona Press), 317

Elsila, J. E., Glavin, D. P., \& Dworkin, J. P. 2010, LPICo, 1538, 5105

Favre, C., Cleeves, L. I., Bergin, E. A., Qi, C., \& Blake, G. A. 2013, ApJL, 776, L38

Gaia Collaboration, Brown, A. G. A., Vallenari, A., et al. 2016, A\&A, 595, A2 Garrod, R. T. 2008, A\&A, 491, 239

Garrod, R. T., \& Herbst, E. 2006, A\&A, 457, 927

Garrod, R. T., Wakelam, V., \& Herbst, E. 2007, A\&A, 467, 1103

Gorti, U., Hollenbach, D., Najita, J., \& Pascucci, I. 2011, ApJ, 735, 90

Harada, N., Herbst, E., \& Wakelam, V. 2010, ApJ, 721, 1570

Hasegawa, T. I., Herbst, E., \& Leung, C. M. 1992, ApJS, 82, 167

Herczeg, G. J., Linsky, J. L., Valenti, J. A., Johns-Krull, C. M., \& Wood, B. E. 2002, ApJ, 572, 310

Herczeg, G. J., Wood, B. E., Linsky, J. L., Valenti, J. A., \& Johns-Krull, C. M. 2004, ApJ, 607, 369

Ioppolo, S., van Boheemen, Y., Cuppen, H. M., van Dishoeck, E. F., \& Linnartz, H. 2011, MNRAS, 413, 2281

Jørgensen, J. K., Favre, C., Bisschop, S. E., et al. 2012, ApJL, 757, L4

Kahane, C., Ceccarelli, C., Faure, A., \& Caux, E. 2013, ApJL, 763, L38

Kuze, H., Kuga, T., \& Shimizu, T. 1982, JMoSp, 93, 248

Kvenvolden, K., Lawless, J., Pering, K., et al. 1970, Natur, 228, 923

Lee, H.-H., Herbst, E., Pineau des Forêts, G., Roueff, E., \& Le Bourlot, J. 1996, A\&A, 311, 690

Lee, H.-H., Roueff, E., Pineau des Forets, G., et al. 1998, A\&A, 334, 1047

Lefloch, B., Bachiller, R., Ceccarelli, C., et al. 2018, arXiv:1803.10292

Lefloch, B., Ceccarelli, C., Codella, C., et al. 2017, MNRAS, 469, L73

Liu, S., Girart, J. M., Remijan, A., \& Snyder, L. E. 2002, ApJ, 576, 255

Loomis, R. A., Cleeves, L. I., Öberg, K. I., et al. 2018, arXiv: 1805.01458

Loomis, R. A., Cleeves, L. I., Öberg, K. I., Guzman, V. V., \& Andrews, S. M. 2015, ApJL, 809, L25

Müller, H. S. P., Schlöder, F., Stutzki, J., \& Winnewisser, G. 2005, JMoSt, 742,215

Öberg, K. I., Guzmán, V. V., Furuya, K., et al. 2015, Natur, 520, 198

Öberg, K. I., Guzmán, V. V., Merchantz, C. J., et al. 2017, ApJ, 839, 43

Parfenov, S. Y., Semenov, D. A., Henning, T., et al. 2017, MNRAS, 468, 2024

Qi, C., Öberg, K. I., \& Wilner, D. J. 2013a, ApJ, 765, 34

Qi, C., Öberg, K. I., Wilner, D. J., \& Rosenfeld, K. A. 2013b, ApJL, 765, L14

Redondo, P., Largo, A., \& Barrientos, C. 2015, A\&A, 579, A125

Rimola, A., Taquet, V., Ugliengo, P., Balucani, N., \& Ceccarelli, C. 2014, A\&A, 572, A70

Salinas, V. N., Hogerheijde, M. R., Mathews, G. S., et al. 2017, A\&A, 606, A125

Schwarz, K. R., Bergin, E. A., Cleeves, L. I., et al. 2016, ApJ, 823, 91

Semenov, D., Hersant, F., Wakelam, V., et al. 2010, A\&A, 522, A42

Semenov, D., \& Wiebe, D. 2011, ApJS, 196, 25

Skouteris, D., Balucani, N., Ceccarelli, C., et al. 2018, ApJ, 854, 135

Trapman, L., Miotello, A., Kama, M., van Dishoeck, E. F., \& Bruderer, S. 2017, A\&A, 605, A69

Wakelam, V., Herbst, E., Loison, J.-C., et al. 2012, ApJS, 199, 21

Walsh, C., Loomis, R. A., Öberg, K. I., et al. 2016, ApJL, 823, L10

Walsh, C., Millar, T. J., Nomura, H., et al. 2014, A\&A, 563, A33

Watanabe, N., \& Kouchi, A. 2002, ApJL, 571, L173

Winnewisser, M., Winnewisser, B. P., Stein, M., et al. 2002, JMoSp, 216 , 259

Yen, H.-W., Koch, P. M., Liu, H. B., et al. 2016, ApJ, 832, 204

Zhang, K., Bergin, E. A., Blake, G. A., Cleeves, L. I., \& Schwarz, K. R. 2017, NatAs, 1, 0130 